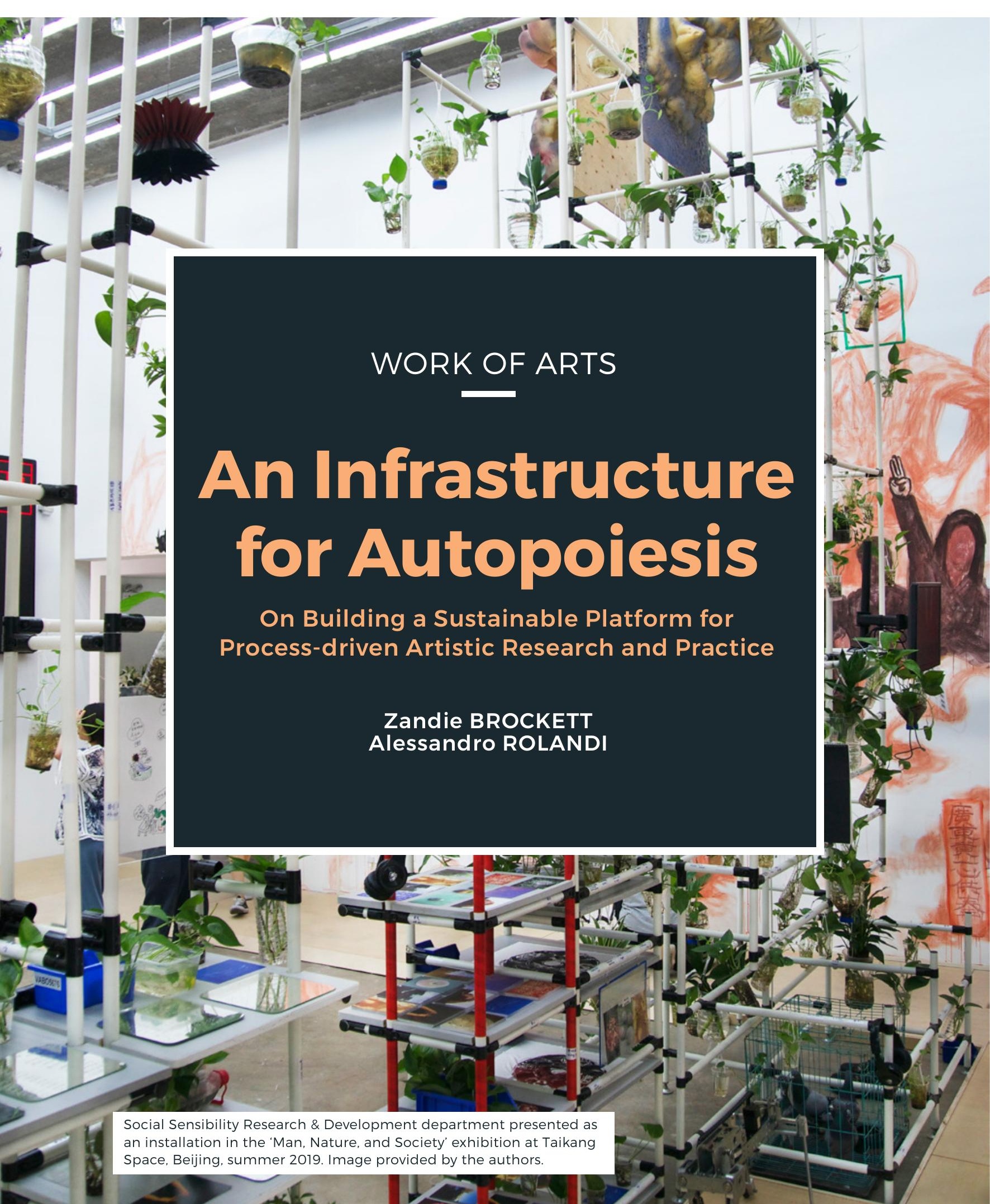




\section{An Infrastructure for Autopoiesis On Building a Sustainable Platform for Process-driven Artistic Research and Practice}

Zandie BROCKETT

Alessandro ROLANDI

Autopoiesis (n) the property of a living system that allows it to maintain and renew itself by regulating its composition and conserving its boundaries.

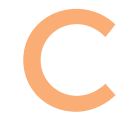

hina's recent economic development policies, including the Belt and Road Initiative (BRI), have served as a poignant reminder of how large infrastructure projects often lose connection with the citizens they are designed to serve. With scale and top-down institutional policies comes the unintended transformation of nuanced lives and communities into mere statistical references. Infrastructure in the sphere of art and culture-existing as a global network of public and private museums, art fairs, biennales, art districts, and creative industry clusters-operates similarly.

Exhibition spaces and museums have long stood as mechanisms that distinguish and construct notions of truth; now, with the shifting tide of consumption and growing demands for participation, much of this cultural infrastructure is evolving into mechanisms for 'edutainment'. Either way, contemporary art-its production, excavated knowledge, and modes of communication-remains guided by institutional logics and market dynamics. How, then, can we remodel cultural infrastructure such that it is a sustainable framework for producing unbiased knowledge given the current economic climate, yet detached from market demands? Further, how could this infrastructure mirror how contemporary societies perform labour and build community?

Perhaps we should think of art not just as objects or remnants of our (material) actions, but also as the processes that are actively retooling the very framework that facilitates the creation of these objects. In this essay, we look at how the Social Sensibility Research \& Development (SSR\&D) department uses process-based art-making and research (i.e. its process) to reimagine how art can serve as a vehicle for the social imagination (i.e. its motive), how this knowledge is transmitted between various social groups (i.e. legitimisation), and how this infrastructure exists in an economically-viable format despite operating at a small, labour-intensive scale (i.e. its economics).

\section{The Social Sensibility Research \& Development Department}

As a site of collective labour and material production, the factory has served a seminal role in the economic, social, and urban development of modern China. Nevertheless, the Chinese factory remains a site of many contradictions. In the reform era, Chinese society subscribed to the idea that 'personal efforts inevitably lead to improvement in one's condition, a value which has become a major mode of legitimation of access to wealth and status' (Florence 2019, 269). But as wealth creation and accumulation has become the primary goal for China's manufacturing and heavy industries, the status and image of the working class as the iconic proletariat has increasingly eroded.

With such a context in mind, in January 2011 Alessandro Rolandi sought to embrace these very contradictions, establishing a platform for open-ended, research-based, artistic projects in 


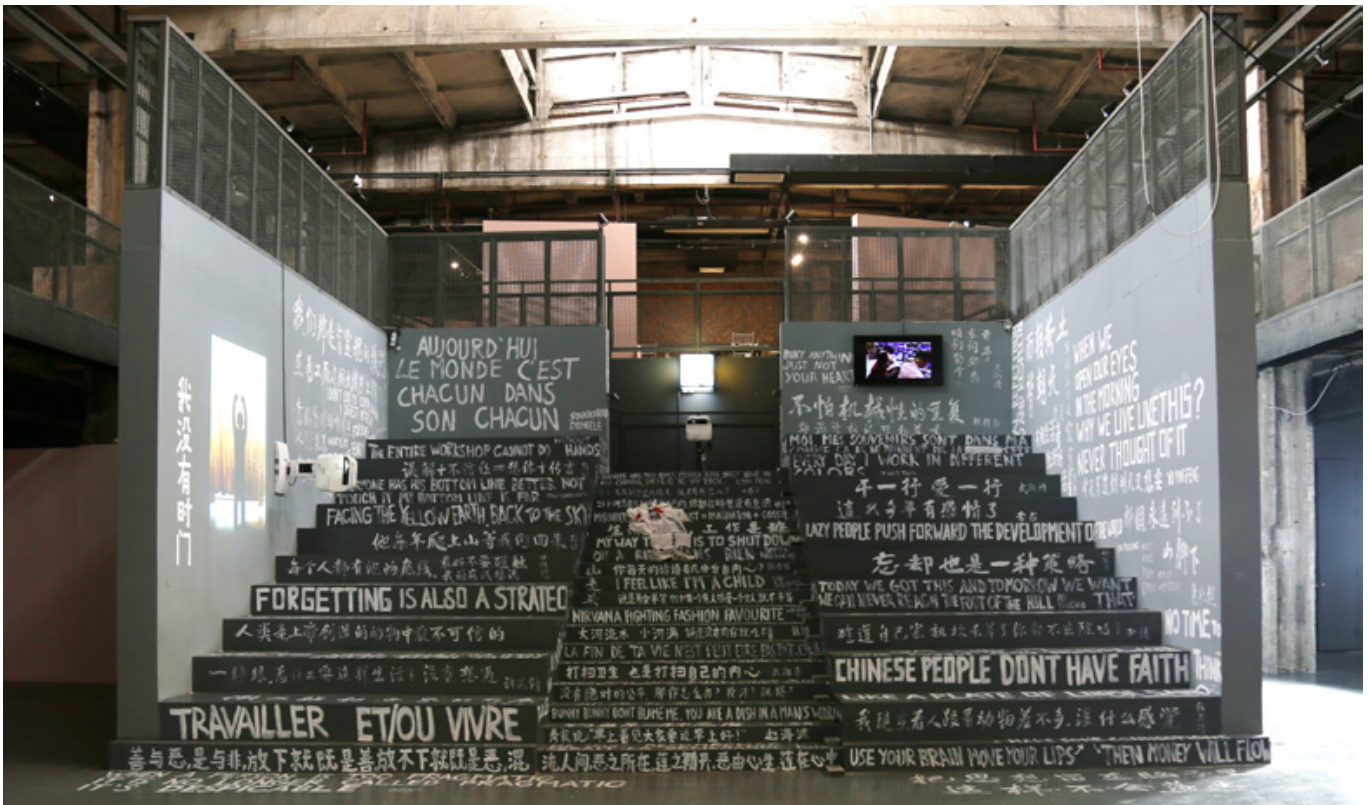

Installation of voices, videos, and written words in English, French, and Chinese from 20 factory workers and employees working at Bernard Controls' China and French factories. Exhibited at Ming Contemporary Art Museum (McaM) Shanghai, fall/winter 2017. Image courtesy McaM.

a privately-held manufacturing company and its factories. SSR\&D is a research and development department at the French-owned-andoperated Bernard Controls actuator-producing plants in Beijing and Paris/Gonesse. Since its establishment, it has served as a platform for the employment of artists who develop long-term, creative, relational, critical, and dialogical activities that engage and collaborate with factory workers. Originally founded as a factory-based residency programme for artists, musicians, curators, scholars, and architects, it has evolved to facilitate the production and exhibition of artworks created by factory workers themselves.

SSR\&D was founded after Rolandi spent nine unpaid months conducting informal research in the Beijing plant of Bernard Controls. During this time, he realised that the company's culture and factory's conditions already provided the workers a certain amount of dignity, respect, and care. This prompted him to introduce something more complex and demandingan art practice. It was then that Rolandi and the factory executives decided to establish the department on the single negotiated condition that all factory workers and company employees have the right to use a portion of their working hours to interact with himself and other invited artists. For these reasons, SSR\&D was founded on sustained practices and long-term commitment existing on a 1:1 scale, which suggests actions should be enacted on a scale between the direct enactor and direct receiver of the practice rather than mediated through spectatorship (Wright 2013). It is an idea and vision where the presence of artists and art practices within an optimised, profit- 


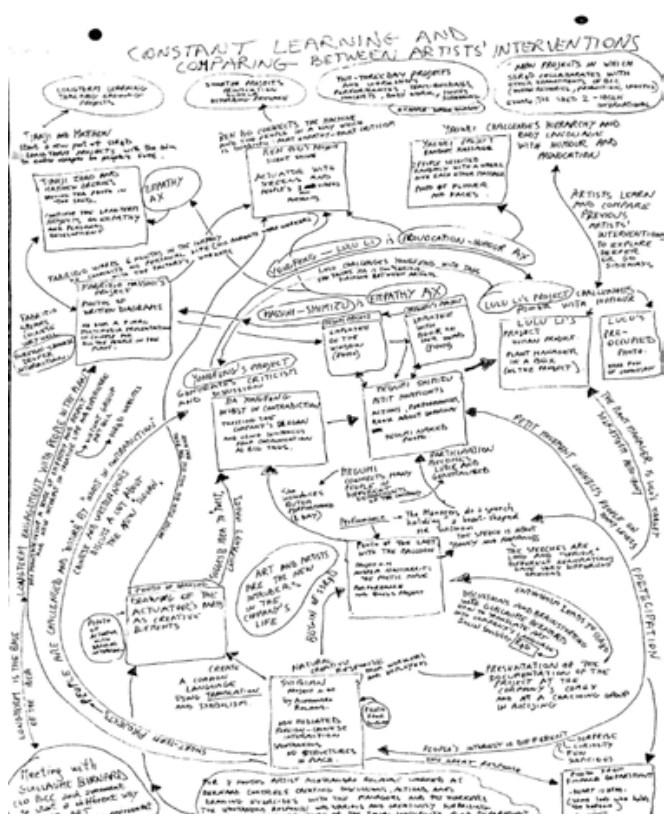

(Left) Map of SSR\&D processes by Alessandro Rolandi for 'Teetering at the Edge of the World' exhibition at Espacio de Arte Contemporáneo, Montevideo, Uruguay, fall 2015. (Right) Installation view of Rolandi's Wall Map in 'Teetering at the Edge of the World'. Images provided by the authors.

driven corporation could trigger unexpected interactions amongst various members of the organisation, and further develop an emotional complexity in the workplace. These 'social sensibilities' (Brockett and Rolandi 2017)-or social skills, emotional capacities, and creative aptitudes that help individuals listen, learn, and adapt to other factory personnel and situations in real time-are cultivated between artist and factory worker, worker and manager, as well as between workers themselves. They attempt to challenge the increased standardisation of human processes and relationships, especially in a manufacturing setting, by fostering new modes of knowledge exchange.

SSR\&D is a flexible form, a living dynamic that provides and defends the openness and freshness of an interactive, artistic framework. It does so by: a) finding inspiration in dialogues

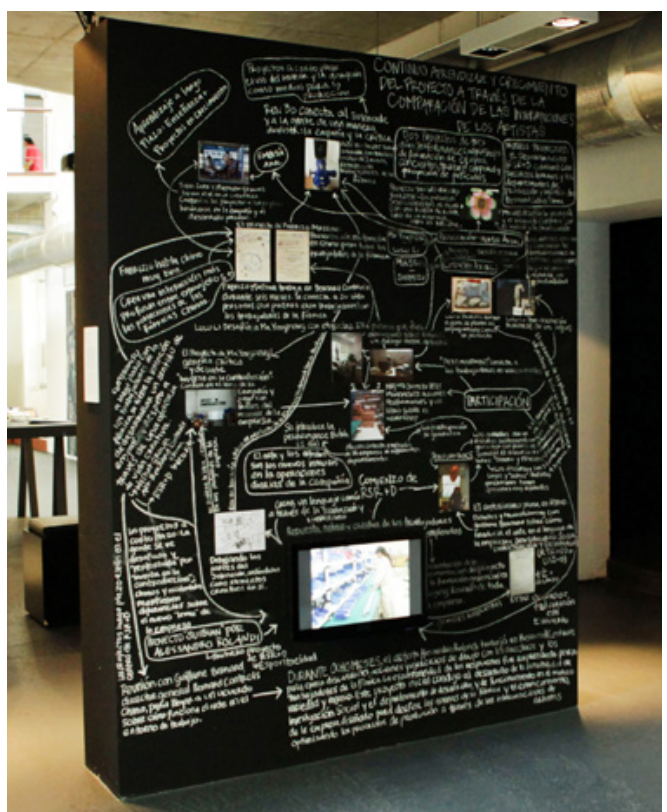

with factory workers and spontaneous collaborations rather than planning ahead; b) abandoning clear ideas of authorship in favour of more complex dynamics of mutual influence; and c) accepting non-professional input, commentary, and critical inquiry as a source of feedback and inspiration. It creates room for thinking that is genuinely creative and spontaneous-which at times results in the creation of art in response to daily working life, and at other times responds to other personal, spiritual, and psychological queries and needs. From indifference to observation, from casual interaction to full intellectual and emotional engagement, the quality of the exchange depends on the emotional and cognitive state of the group. 


\section{Process}

The SSR\&D department operates as an integrated unit within the Bernard Controls company. The 'research and development' component of its name reflects the practicebased nature of the department's work, while contextualising its function within operations, rather than in the realms of human resources or corporate social responsibility. The function and mission of the department also informs its structure. The department currently consists of a team of three artists hired with a full-time working contract, salary, and health insurance: two in Beijing (Alessandro Rolandi and Tianji Zhao) and one in Paris (Blandine de la Taille). Both units remain in constant communication as they attend their respective Beijing and Paris/ Gonesse factories on a regular weekly basis. External artists are invited for two to threemonth-long residences, of which there are no more than four per year per factory. Within this unique opportunity to work within a corporate entity and in a fully-operating factory, SSR\&D artists are allowed to engage with the company, its procedures, equipment, space, workers, administrators, and staff. Artists interact with employees through discussion and mutual exchange of ideas and materials in an informal and impromptu manner. Over the past eight years, as SSR\&D has brought 43 artists and some 30 scholars and industry professionals into the factory, a wide source of knowledge and practices have emerged.

For each visit from an artist, arrival at the factory is between 10 and llam. A lunch is served for department and visiting artists in the conference room, which also doubles as the SSR \&D office. At $2 \mathrm{pm}$, once the workers return from their lunch break, and until $5 \mathrm{pm}$, when the working day concludes, SSR\&D artists are allowed onto the factory floor and into the offices. It is here, with the mere physical presence of the artist, that the process-based research begins. Roaming with books, physical artworks, and videos-on-phones in hand and ready to share, each artist explores different approaches that allow them to establish a personal connection with factory workers. Often inquiring about the worker's life and work situation, the artists use these materials as tools to deliver stories about how they or other artists have dealt with similar ideas and situations. Each interaction attracts voluntary degrees of closeness, collaboration, and criticality from the workers, while providing the artist an opportunity to develop an awareness about the dynamic's natural flow, an acute attention to the subtly of response, and a patience for awkwardness during the exchange. Each of these skills become 'social sensibilities', or when employed, methodologies for soliciting participation, exchange, and negotiation.

Over time, common interests or past experiences are revealed and through continued conversation, these ideas evolve into artistic projects. Some are didactic or collectively participatory, while others are centred around craft, object-making, and sound-based experimentation.

\section{Motive}

In this hyper-connected world propelled by, but also overloaded with, information exchange, the nature of learning and work are dramatically changing. Through processes of storytelling-that is presence-driven connection and exchange-we find an analogic, flexible, informal, and creative way of engaging with each other, finding the commonalities in our collective existence. Through anecdotes, we might find a shared social fabric, criticality, and memory that prevent technological and economic infrastructures from reducing social life to solipsistic feedback loops ruled by standardised machines and optimised systems. With story-based exchange, we give birth to unexpected alliances that establish undercurrents of knowledge production and where art becomes a catalyst for unusual relationships and strategies that address and express personal and collective issues. These 


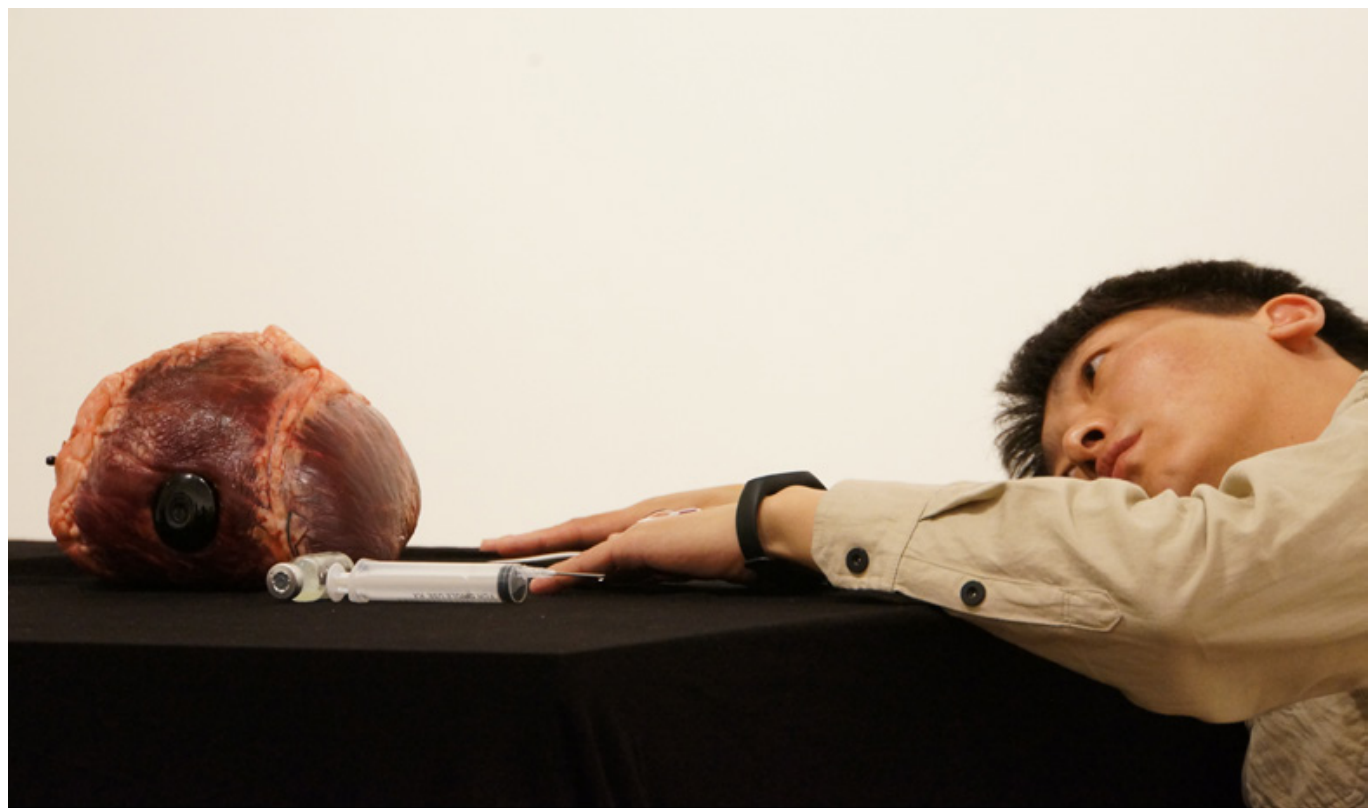

Bernard Controls China factory worker, Wei

Chengcheng, performing his 'Sensitive Heart' artwork at Yang Art Museum (YAM), Beijing, April 2017. Image provided by the authors.

stories are activated as 'new material' for use in artworks. In doing so, they distil, transform, and activate the mundaneness of life and the vernacular of labour into a dynamic that elevates and empowers the experience, the individual, and their skills.

While fundamental to the practice, these relationships are not themselves the main objective of the interactions between artist and worker. Rather, the department understands that the interactions are of bidirectional influence and that this more subtle, selfadjusting, and intuitive form of exchange enables the negotiation of taste (Mörsch 2011). Within this process of discussing, exchanging, spontaneously reacting, and collectively deciding, the multifarious 'tastes' found within social life-as conditioned by various ethnicities, genders, classes, and ages-are mediated. This process allows individuals to gain new experiences and insights through which they can perceive the realities of the 'Other'.

\section{Legitimisation}

The department facilitates the production, display, documentation, and promotion of all artworks created by resident artists, as well as those produced by workers themselves. It further fosters relationships between itself, galleries, and museums in the art world at large to establish opportunities for the exhibition, presentation, and in a way, the external legitimisation of artworks created by employees of the factory. The presence of non-professional artists (i.e. factory workers, managers, and employees) and their work 
exhibited in galleries and museums, as well as during lectures and workshops, is not intended as a provocation or confirmation of an unusual art education. Rather, it is meant to extend the dialogue about the role of artists in society and the potential value of art when dissociated from the commodity and spectacleoriented dynamic of an elite market system. SSR\&D's presence in art institutions intends to ask fundamental questions like: where is art conceived and made, and where could it be conceived and made? By whom? For what purpose? Who is the audience? What is art's impact on reality and how can we measure it? How do we value artwork and how do we value art practice, or the process by which artwork is made?

Most importantly, the SSR\&D department also internally validates and legitimatises alternative forms of knowledge production and learning with a gravity akin to formal institutions. With its one founding conditionworkers have the freedom to allocate a portion of their labour time to engage with invited artists-the SSR\&D department produces infrastructure that recognises the value of casual interaction and promotes engagement in artistic labour. Through this process of discussing memories and daily life, anecdotes are exchanged but also creatively valued and validated. The department establishes its credibility in recognising forms of cultural knowledge found in aesthetic skills, nonlinear thinking, and non-verbal languages that negotiate and address the tensions, contradictions, and impasses between people of different specialities and levels of seniority.

By allowing this, Bernard Controls implies that exploring artistic labour and articulating intellectual, psychological, and emotional curiosities are of value equal to the labour performed by full-time employees, on the factory line or at their desks. By paying both invited artists and employees to make art, Bernard Controls also-much like the museum's relationship with objects from, for instance, the colonised world-legitimises the knowledge produced by the 'Other'. These layers of information are non-hierarchical and are not systematically referenced, as is with formal curricula or corporate policies. Rather they are organically circulated and shared, thereby establishing the intimacy necessary in alternative modes of corporate management and negotiation. SSR\&D shifts the geography of knowledge production within the company by valuing information, skills, and stories that arise from non-academic systems.

\section{Economics}

The department together with Bernard Controls has created an economic model that provides steady funding to artists as researchers, not paid out in a sporadic or competitive manner but rather as a monthly salary for a properly hired R\&D professional. The remuneration, conceived of as research fees, pays for the time artists spend engaging workers in activities and dialogue, rather than as fees for the 'production' or purchase of an artwork. This economic model invites us to consider the future potentiality of an artist being paid for engaging with complex and experimental field research, instead of depending on the commission or sale of artworks. As such, an artist can secure financial stability with their long-term commitment to researching and developing a single project. Furthermore, instead of investing in the acquisition of artworks, capital-in this instance, from Bernard Controls' operational budget-can be allocated towards improving conditions for producing artworks. This economic model prioritises creative actions and thinking, the role of artists within society, and more complex logics of cultural production and distribution that escape the gravity of a market economy.

Importantly, all artworks created in the factory and under the supervision of the department belong to those who created them. Unlike corporate policies mandating everything produced at the company remains 


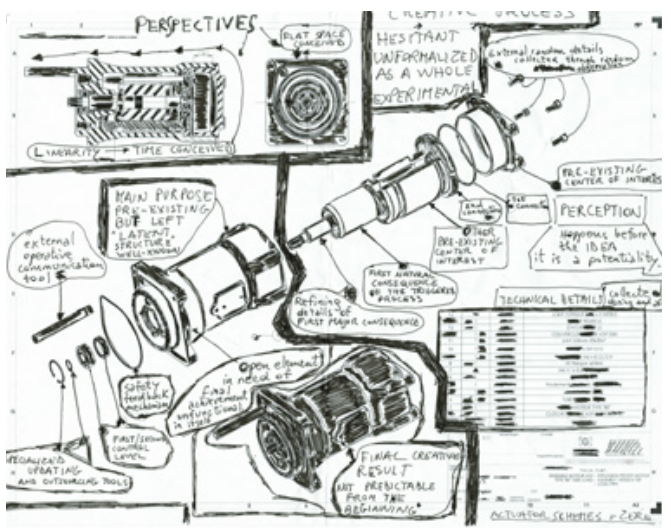

'Techno Poetic Drawing' by Alessandro Rolandi on blueprints for factory-made actuator parts, 2011/2012. Image provided by the authors.

property of that entity, the authorship, physical object, its intellectual property, and value of each artwork is transferred to its creator(s).

While some may ask what the factory receives in return, a subtler reply might be that the organisational culture shifts towards a more collaborative dynamic. SSR\&D pushes Bernard Control managers to think and behave in ways that allow 'non-essential' personnel to work within the factory space. During the working day, SSR\&D shifts standardised managerial practices away from hierarchical, order-giving strategies, and towards ones that attend to, and are patient with, the process of negotiating human dynamics.

Commensurate with local incomes, these salaries and stipends are benchmarked by employer standards. Most importantly, this research position is conceived so that artists' basic needs like health insurance, rent for a studio/home in the country of residence, and even some materials for their own studio practice can be afforded. A model of this kind supposes that artists could spend roughly half of their career (depending on interest levels) being remunerated for simply being artists and conducting research in a participatory social framework.

\section{Potentiality}

As SSR\&D moves towards its ten-year anniversary, we ask how its model might situate itself within different factories across China, if not companies from different sectors and around the world. Is the practice that SSR\&D puts forth one that could be incorporated into organisations of larger dimensions? Is it a model that might stimulate the social imagination of workers in factories or companies with highly automated processes, and thus prove as another approach to shape and circulate artistic, cultural, even organisational knowledge? Is it a model that is unique to China's labour context, given the country's State-let market economy that enables the rise of innovative infrastructural forms and the rapid prototyping needed to build these models?

By embedding artists into a factory and within the hours of a working day, the SSR\&D department develops organisational infrastructure that activates time and a flexible creative arena for the exploration of ambiguity within a streamlined manufacturing context. This infrastructure does not maintain a logic of the recreational, commercial, or cultural, but rather of daily work and life-spaces where the majority of human time is spent and patterns of thought and action are habituated. As artists coopt time from the workers in between their paid tasks, the informal exchanges and discussions of personal interests redefine and reposition the importance of creative and expressive individuals. It is SSR\&D's legitimised space that maintains distance from a defined purpose that allows voluntary spontaneities and flexible sensibilities to organically adapt to ambiguity. It is an infrastructure that is not forced upon its users, but rather values and incorporates the multiplicity of knowledge, forms of creative expression, and styles of learning and engaging into its pillars. 